\title{
Low Dose Computed Tomography (LDCT) Versus Chest X-ray in Chronic Smokers- Can LDCT be Used as a Motivation to Quit Smoking?
}

\author{
Authors \\ Dr Tosha Jatin Desai ${ }^{1}$, Dr Ekta Vinubhai Vadher ${ }^{2}$, Dr Nandini Umesh Bahri ${ }^{3}$ \\ Corresponding Author \\ Dr Tosha Jatin Desai \\ Kanti', 102/C Mahavir C society, Near Shivam Paan Center, Jamnagar, Gujarat, India
}

\begin{abstract}
Background- Smoking is one of the biggest health threats to mankind. Early diagnosis of pulmonary changes induced by smoking can help in timely intervention and prediction of course of smoking-induced diseases.

Aim-The aim of this study was to determine the relative prevalence of various common radiological findings associated with smoking and compare their detection on digital chest X-ray and Low Dose Computed Tomography (LDCT)

Materials and Methods-Chest X-ray PA view and LDCT scan of 50 chronic symptomaticlasymptomatic male smokers were analysed over a period of 1 year from February 2016 to February 2017.

Summary- Chest X-ray was less sensitive in detecting early emphysematous changes, small pulmonary nodules and minimal fibro-bronchieactatic changes. In our study, spectrum of respiratory findings in smokers on LDCT included bronchial thickening (68\%) emphysema (66\%), interstitial thickening (61\%), pulmonary micro- and macro- nodules, air space opacification, bronchieactasis, mediastinal lymphadenopathy and ground-glass opacities. The sensitivity of LDCT in diagnosing malignant lesions was $88.4 \%$ and speceficity was $82.5 \%$. The sensitivity of Chest Xray in diagnosing malignant lesions was $50 \%$ and specificity was $87.5 \%$. Conclusion- LDCT diagnoses malignant lesions much earlier and provides a better graphical picture of the pathology under study to young and middle aged smokers willing to quit smoking. Since the baseline risk of lung cancer development is small and the dose exposure in a single LDCT scan is well below the annual adult radiation dose limit, the risk-benift ratio is very favourable.
\end{abstract}

Keywords-smoking, smoking-induced diseases, Chest X-ray, LDCT, risk-benifit ratio.

\section{Introduction}

The tobaccoo epidemic is one of the biggest public health threats the world has ever faced. Nearly $80 \%$ of more than 1 billion smokers worldwide live in low-and middle income countries, where the burden of tobacco-related illnesses and death is heaviest. Smoking is responsible for more than $80 \%$ of all lung cancers worldwide.

Smoking related diseases can be classified asInterstitial lung diaseases (Respiratory bronchiolitis,
Desquamative interstitial pneumonia, Pulmonary Langerhans histiocytosis, Acute eosinophillic pneumonia, Combined pulmonary fibrosis and emphysema), Neoplasms (Lung cancer, Tracheal tumours) and Chronic Obstructive Pulmonary Disease (Emphysema, Chronic bronchitis, Constrictive bronchiolitis). ${ }^{2}$

The routine protocol of most clinicians in a patient presenting with respiratory complaints is to order a preliminary Chest Xray PA view. ${ }^{3}$ However, since it 
is a two-dimensional imaging modality, much information is lost due to overlap of organs, patient motion and inadequate breath-hold. Hence, early and subtle changes are often missed and the patient is falsely convinced of being disease-free. ${ }^{4}$

The role of LDCT in lung cancer screening is established by NLST (National Lung Screening Trial). ${ }^{5}$ Screening is the testing of an individual who is at risk for a disease, but who does not exhibit the signs of the disease. ${ }^{6}$ It involves scanning of an individual with average dose of 1.5 to $3.4 \mathrm{mSv}$ (further impovements have led to further dise reduction upto a level of $0.2 \mathrm{mSv}$ ) which is 4 to 12 times more than Chest X-ray and 5 times less than conventional CT chest. $^{7}$

LDCT is comparable in sensitivity and specificity of lung nodule detection with conventional chest CT.6 In addition it offers all other advantages of a CT scan such as three dimensional reconstruction, single breath-hold and exploits the inherent high contrast of thoracic structures. However, it suffers from over-diagnosis and a high false positive rate. ${ }^{8}$

Since cigarette smoking results in a focal accumulation of macrophages within the walls of respiratory bronchioles and adjacent alveoli, the common finding on chest X-ray is of "dirty chest", an overall increase in non-specific lung markings. Remy Jardin et $\mathrm{al}^{9}$ found that the morphological substrate of cigarette smoking- induced changes in chest radiography were parenchymal micro-nodules and intra-lobular opacities. Destructive process such as emphysema results in increased parenchymal lucency with preserved or increased lung volumes. In a study by $\mathrm{J}$ Kirchner et al, ${ }^{9}$ there was a significant positive correlation between the increase of overall lung markings on chest radiography and the cigarette consumption quantified by pack-years. There is a positive linear correlation between bronchial wall thickening and intra-lobular opacities as seen in CT with the increase of overall lung markings on radiography.

\section{Aims}

- To study the effects of smoking on respiratory system.
- To determine the relative prevalance of various common radiological findings associated with smoking and to corelate it with the amount of cigarette consumption measured in pack-years.

- To study and compare the findings on two modalities - Digital X-ray Chest and LDCT.

- To calculate the risk-benefit ratio of using either modality in terms of its sensitivity, specificity and dose of ionizing radiation.

- To target especially young chronic smokers and explain them the effects of smoking to their lungs, the importance of early diagnosis and motivate them to quit smoking.

\section{Materials and Methods}

The present study was conducted in a tertiary care center with 50 subjects from February 2016 to February 2017.

\section{Description of tools:}

- Digital X-rays (PA view of chest) procured through SIEMENS mm-10 160mAs Xray (HF) machine

- Non-contiguous Low dose computed tomography axial cuts with reformation procured through 16 slice MDCT machine (GE healthcare).

\section{Inclusion Criteria}

1. Age : 35 years or more

2. Sex : male/female

3. Active smoking history of 10 years or more (present or past).

4. Symptomatic/asymptomatic.

\section{Exclusion criteria}

1. Known case of primary/secondary lung cancer or any chronic lung disease.

2. No previous CT imaging for lung pathology.

3. Scanning protocol

\section{For conventional radiography}

-kvp 40-60

-mAs 20-30

-Exposure dose on an average $0.02 \mathrm{mSeivert}$

For low dose computed tomography:

- Number of detectors: 16

$-\mathrm{kVp}: 80$ 


\section{JMSCR Vol||05||Issue||06||Page 23166-23172||June}

- $\mathrm{mA}:$ 30-50 CT-AEC (SD = 70)

- Seconds/rotation : 0.75

- mAs : 22.5-37.5

- Pitch factor : 1.438

- Collimation : $1 \mathrm{~mm} \times 16$

- Reconstruction slice thickness (mm) : $1.5 \mathrm{~mm}$

- Slice interval $(\mathrm{mm}): 3$

- Lung field : 1600/-600

- Mediastinal : 400/35

- Exposure dose(range): 1.5 -3.4 mSv

- Area covered: From apex of lung to diaphragm.

The number of true positives and negatives were calculated for both Chest Xray and LDCT based on clinico-histopathological correlation. Based on it, the sensitivity and specificity of both diagnostic modalities were calculated. Chi-square test was applied for association of amount of cigarette consumption with pack-years with value of $\mathrm{X}^{2}=$ 4.23, $\mathrm{p}$ value $<0.05$ and degrees of freedom: 1 .

\section{Observations}

The distribution of various pulmonary findings, relative prevalence, detectability on Chest x-ray and LDCT is as described below-

Table $1 \%$ Distribution of smokers according to pack-years

\begin{tabular}{|l|c|c|}
\hline $\begin{array}{l}\text { Smoking in } \\
\text { pack-years }\end{array}$ & $\begin{array}{c}\text { No. of patients } \\
(\mathrm{n}=50)\end{array}$ & \%Percentage \\
\hline $11-15$ & 6 & 12 \\
\hline $16-20$ & 6 & 12 \\
\hline $21-25$ & 11 & 22 \\
\hline $26-30$ & 11 & 22 \\
\hline $31-35$ & 8 & 16 \\
\hline $36-40$ & 6 & 12 \\
\hline $41-45$ & 2 & 4 \\
\hline
\end{tabular}

Majority of smokers had a smoking exposure of 2130 pack years.

Table $2 \%$ Distribution according to symptoms

\begin{tabular}{|l|c|c|}
\hline Symptom & $\begin{array}{c}\text { No.of patients } \\
(\mathrm{n}=50)\end{array}$ & $\%$ Percentage \\
\hline Dry cough & 34 & 68 \\
\hline Cough with expectoration & 17 & 34 \\
\hline Dyspnoea & 32 & 64 \\
\hline Hemoptysis & 9 & 18 \\
\hline Weight loss & 8 & 16 \\
\hline Fever & 1 & 2 \\
\hline Chest pain & 2 & 4 \\
\hline
\end{tabular}

Most common presenting complaint was dry cough followed by dyspnoea.
Table $3 \%$ Distribution of common radiological findings

\begin{tabular}{|l|c|c|c|c|}
\hline Radiological finding & \multicolumn{2}{|c|}{$\begin{array}{c}\text { Chest X-ray(n=50) } \\
\text { No. }\end{array}$} & \multicolumn{2}{c|}{ LDCT(n=50) } \\
& No. & 68 \\
\hline Bronchial thickening & 28 & 56 & 34 & 60 \\
\hline Interstitial thickening & 20 & 40 & 30 & 66 \\
\hline Emphysema & 21 & 42 & 33 & 50 \\
\hline Pulmonary nodules & 16 & 32 & 25 & 18 \\
\hline $\begin{array}{l}\text { Areas of air space } \\
\text { opacification }\end{array}$ & 7 & 14 & 9 & 10 \\
\hline Consolidation & 6 & 12 & 5 & 32 \\
\hline $\begin{array}{l}\text { Ground-glass } \\
\text { opacities }\end{array}$ & 0 & 0 & 4 & 4 \\
\hline $\begin{array}{l}\text { Mediastinal } \\
\text { lymphadenopathy }\end{array}$ & 10 & 20 & 16 & 2 \\
\hline Pleural effusion & 2 & 4 & 2 & 0 \\
\hline
\end{tabular}

The three most common findings were the presence of bronchial thickening, emphysema and interstitial septal thickening.

Figure $1 \%$ of prevalance of bronchial thickening on Chest X-ray and LDCT

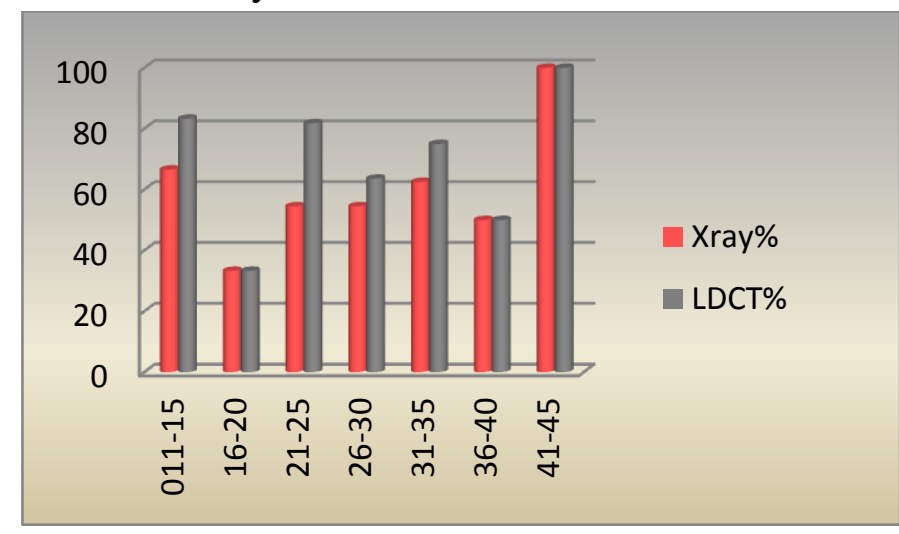

There was no significant difference between moderate and severe consumption of smoking as regards to prevalence of bronchial thickening on chest X-ray and LDCT.

Figure $2 \%$ Distribution of different types of empysema acording to pack years

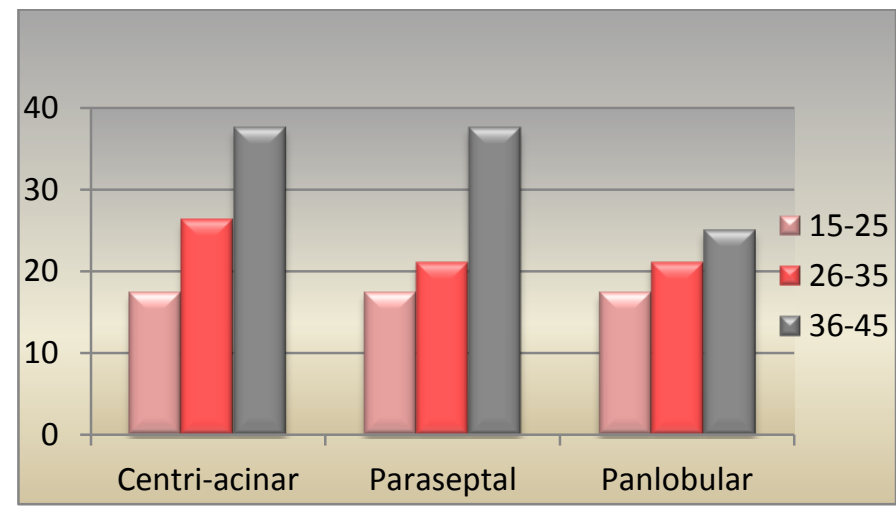

All types of emphysema were strongly related to smoking with a positive correlation to amount of cigarette consumption measured in pack-years. 


\section{JMSCR Vol||05||Issue||06||Page 23166-23172||June}

Figure $3 \%$ Distribution according to number, size and consistency of nodules

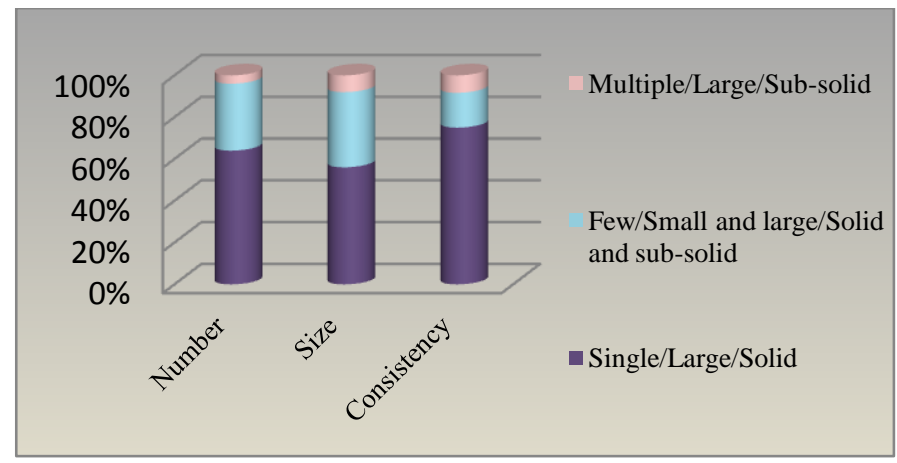

Figure $4 \%$ Distribution of benign and malignant findings on Chest X-ray and LDCT

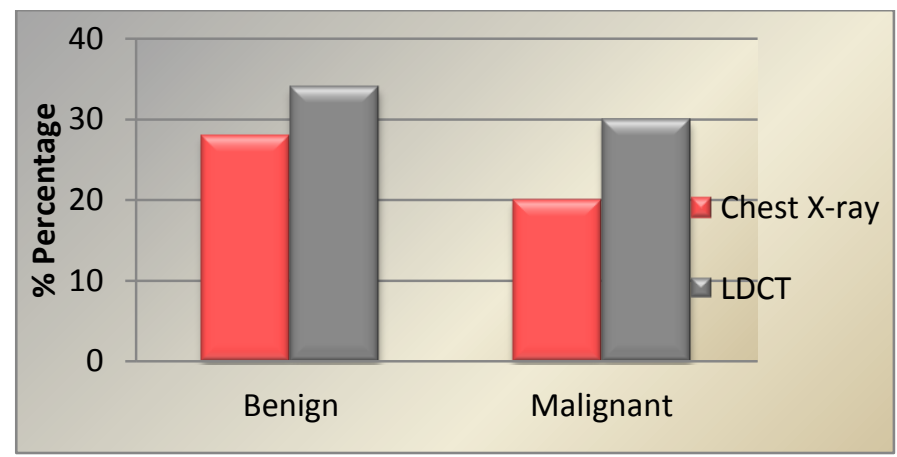

The proportion of benign lesions in smokers was higher than malignant lesions by diagnosed by both Chest X-ray and LDCT. LDCT could diagnose more benign and malignant lesions than chest X-ray.

Figure 5 Comparison between sensitivity and specificity of chest X-ray and LDCT in diagnosing malignant lesions

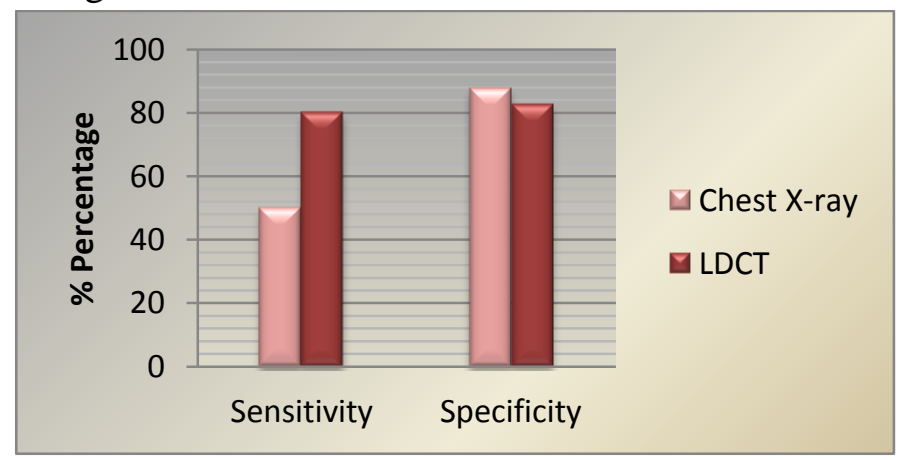

LDCT was sensitive in diagnosis of malignant lesions as compared to Chest X-ray, however, its specificity was slightly lower as compared to chest X-ray. With modern multi-detector CT, pulmonary nodules are detectable at a size of less than $2 \mathrm{~mm}$, however, most of them are benign giving rise to false positive results
Table 4 Dose exposure in chest Xray and LDCT

\begin{tabular}{|l|c|c|c|}
\hline $\begin{array}{l}\text { Dose range }(\mathrm{X}- \\
\text { ray }(\mathrm{mSv})\end{array}$ & No.of patients & $\begin{array}{c}\text { Dose range } \\
(\mathrm{LDCT})(\mathrm{mSv})\end{array}$ & No.of patients \\
\hline 0.18 & 7 & 1.4 & 1 \\
\hline 0.19 & 7 & 1.5 & 10 \\
\hline 0.2 & 11 & 1.6 & 9 \\
\hline 0.21 & 7 & 1.7 & 8 \\
\hline 0.22 & 11 & 1.8 & 11 \\
\hline 0.23 & 4 & 1.9 & 9 \\
\hline 0.24 & 3 & 2 & 2 \\
\hline Total & 50 & - & 50 \\
\hline
\end{tabular}

Figure 6 Multiple nodules with interstitial septal thickening

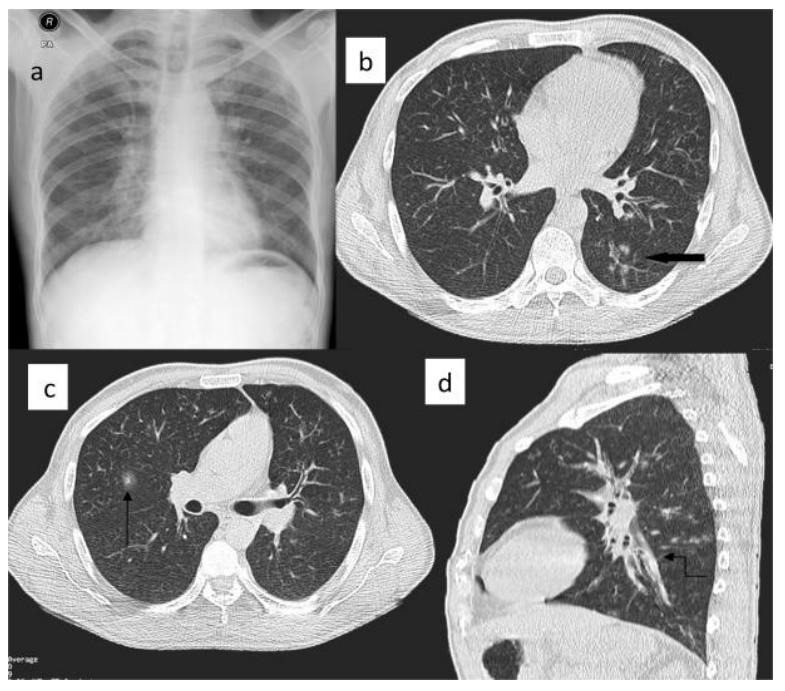

45 year old smoker with dry cough, chest Xray (figure a) appears unremarkable. LDCT axial scans (figures b and c) show few solid micro-nodules in superior segment of left lower lobe in perilymphatic distribution (thick arrow) and a sub-solid nodule in anterior segment of right upper lobe (thin arow). Coronal reformatted image (figure d) shows peri-bronchovascular cuffing (elbowed arrow).

Figure 7 Multiple parenchymal nodules with cavity formation

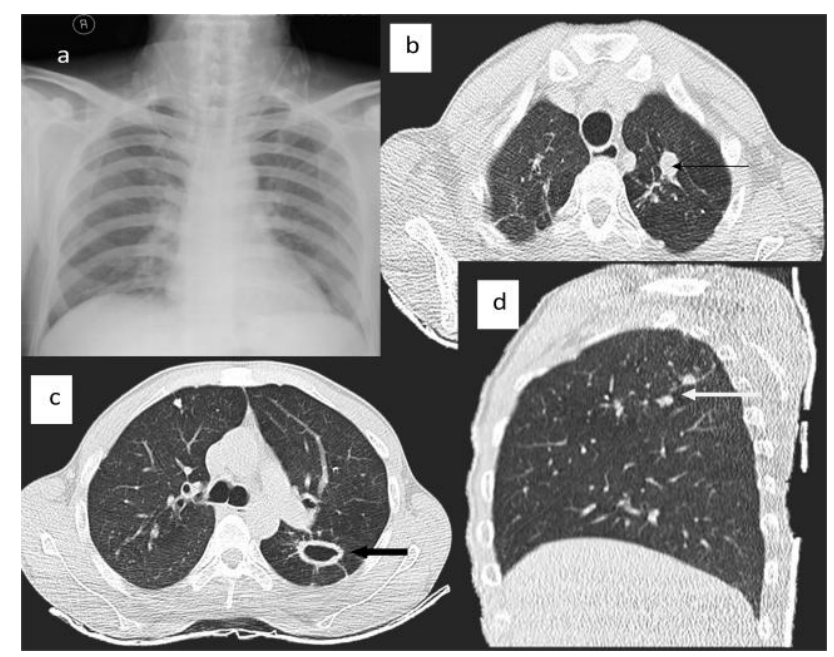


40 year old male smoker with complaints of dry cough. Chest X-ray (figure a) appears unremarkable. LDCT images (figures $b$ and $d$ ) show few irregularly marginated solid nodules(thin arrows) in random distribution in apicoposterior segment of left upper lobe, (figure c) shows a cavitory lesion with outer irregular and inner smooth margin with perilesional interstitial thickening(thick arrow)in apico-posterior segment of left upper lobe.

Figure 8 Bullous emphysematous changes

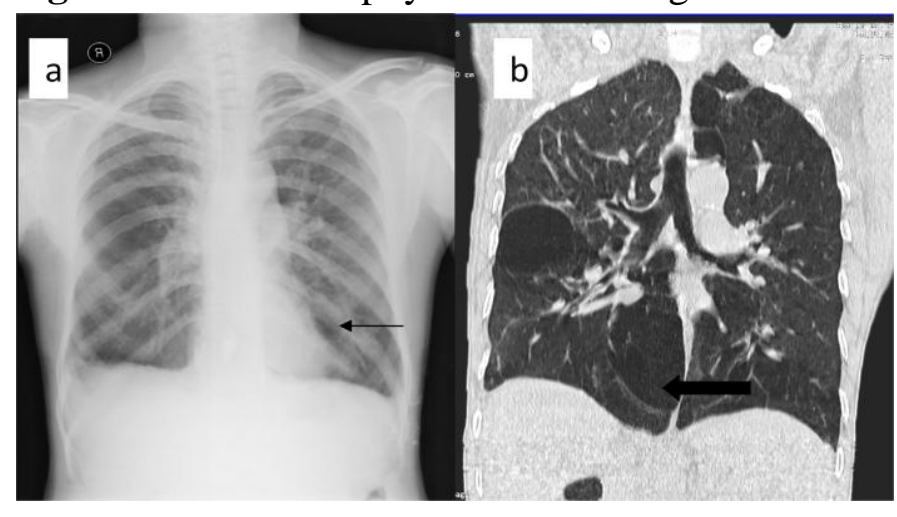

38 year old male smoker with recurrent breathlessness. Chest Xray (figure a) shows patchy areas of radiolucency in bilateral lungs (thin arrow). LDCT coronal image (figure b) shows moderate centri-acinar and panacinar emphysema with bulla formation(thick arrow).

Figure 9 Solitary pulmonary nodule, malugnant etiology

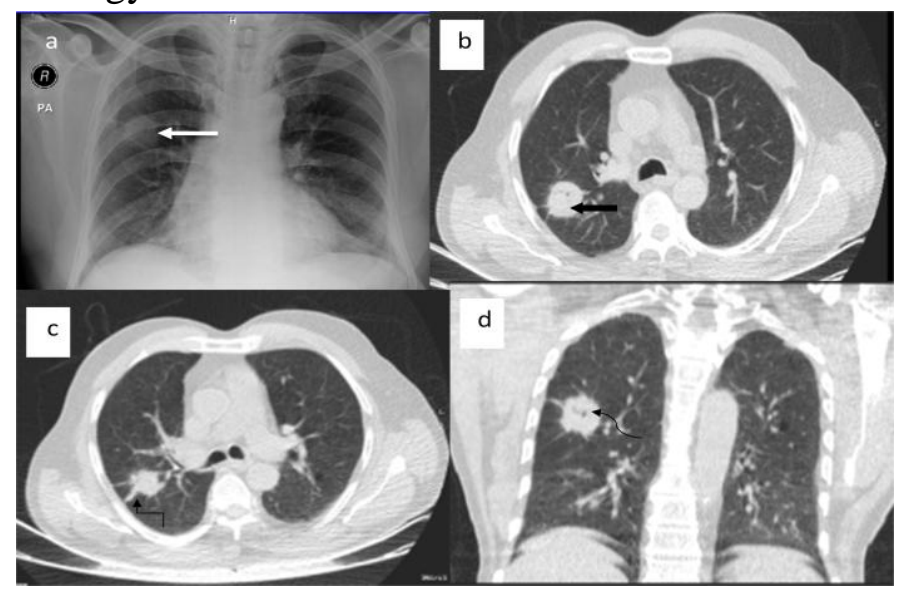

50 year old with hemoptysis. Chest X-ray (Figure a) shows a rounded soft tissue radio-opacity in right upper lung zone. LDCT images (figures b, c and d) show a spiculated marginated nodule (thick arrow) with perilesional nodular thickening (elbowed arrow) suggestive of lymphangitic spread and central necrosis (curved arrow).

\section{Discussion}

In our study 50 chronic smokers were subjected to both chest X-ray and LDCT according to standard protocols to evaluate and compare the spectrum of respiratory findings associated with smoking. Various recent studies on epidemiology of smoking related diseaes, radiological patterns, corelation between tobacco burden and radiographic appearances, dose exposure were kept as reference. ${ }^{10}$

Majority of smokers (44\%) had a smoking exposure equivalent to 21-30 pack-years and all smokers were males reflecting that smoking habit is widely prevalent among Indian men than women. No female smoker attended our institution during the study period fulfilling the inclusion criteria; hence the findings were completely obtained on male subpopulation. Majority of smokers were active smokers $(90 \%)$.

The most common presenting symptom was dry cough $(68 \%)$ followed by breathlessness.(64\%). The spectrum of respiratory findings in smokers included bronchial thickening, emphysema, interstitial thickening, pulmonary micro and macro-nodules, areas of air space opacification, bronchieactasis, mediastinal lymphadenopathy and ground-glass opacities. No significant correlation was found between lung cysts, consolidation, parenchymal fibrosis, honeycombing, pleural effusion and smoking. $(\mathrm{p}<0.001)$

Only a small proportion of LDCT scans in smokers were completely normal. Chest Xrays were normal in more number of patients $(36 \%)$ as compared to LDCT(12\%).LDCT was able to detect subtle findings in $24 \%$ of patients whose X-rays were considered normal.

The most common finding in smokers was the presence of bronchial thickening $(68 \%)$ on both Chest X-rays and LDCT. Bronchial thickening was not associated with the amount of cigarette consumption measured in pack-years. It was present in varying proportions in mild, moderate and heavy smokers. 
The second most common finding on both Chest Xray and LDCT was the presence of Emphysema (62\%). All types of emphysema were associated with smoking. The prevalence of emphysema and its severity increased with the increase in cigarette consumption measured in pack-years.LDCT $(36 \%)$ was superior in diagnosing early emphysematous changes as compared to X-rays(21\%).

The third most common finding was increased interstitial opacities on LDCT or increase in nonspecific parenchymal markings on Chest X-ray.The prevalence of interstitial opacities showed a positive corelation with cigarette consumption measured in pack-years. $\left(X^{2}=5.6, \mathrm{p}<0.01\right)$.

Pulmonary micro-nodules showed a positive corelation with smoking. Most of the nodules detected on Chest Xray and LDCT were of benign etiology. LDCT could diagnose more benign and malignant nodules/areas of air space opacification as compared to Chest X-rays. However, LDCT had a slightly higher false positive rate, i.e, few malignant nodules diagnosed on LDCT turned out to be of benign etiology.

LDCT was sensitive in diagnosis of malignant lesions as compared to Chest X-ray, however, its specificity was slightly lower as compared to chest X-ray.

The effective radiation dose was approximately 7 times higher in LDCT as compared to Chest Xrays. However, LDCT diagnosed potential malignant lesions much earlier and provided a better graphical picture of the pathology under study. Since the base-line risk of lung cancer development is small ${ }^{11}$ and the dose exposure in a single LDCT scan is well below the annual adult radiation dose limit, the riskbenefit ratio in employing LDCT over chest X-rays is very favourable.

Early diagnosis and evidence based-counselling ultimately leads to improved patient outcome. Young and middle-aged smokers were explained the damage smoking had done to their lungs, further health implications if they continued to smoke and hence effectively dissuaded to quit smoking.

\section{CONCLUSION}

For a young to middle aged chronic smoker LDCT can serve as a convenient diagnostic modality to evaluate early pulmonary changes induced by smoking, when X-ray findings are non-specific. Further studies are required to establish cost-benefit analysis and impact on quality of life when LDCT is used as a patient counselling tool.

\section{ACKNOWLEGMENTS}

We are thankful to Dr.H.P.Parekh (M.D, Professor), Dr.S.L.Chudasama (M.D, Associate Professor) for their inputs on the subject and to Mr.Vimal and Mr.Mahendra, our skilled technician staff.

\section{REFERENCES}

1. World Health Organisation. Tobacco [Internet]. Geneva: World Health Organisation; 2017 May [cited 2017 June 1]. Available from:

http://www.who.int/mediacentre/factsheets/f s339/en/

2. Galvin JR, Franks TJ. Smoking-related lung disease. Journal of thoracic imaging. 2009 Nov 1;24 (4):274-84.

3. Mayo clinic.Chest X-rays.[Internet]2014 May [cited 2017 June 1]. Available from: http://www.mayoclinic.org/testsprocedures/chest-Xrays/basics/definition/prc-20013074

4. Cardinale L, Volpicelli G, Lamorte A, Martino J, Veltri A. Revisiting signs, strengths and weaknesses of Standard Chest Radiography in patients of Acute Dyspnea in the Emergency Department. Journal of thoracic disease. 2012 Aug;4 (4):398.

5. National Lung Screening Trial Research Team. The national lung screening trial: overview and study design. Radiology. 2011.

6. Sharma D, Newman TG, Aronow WS. Lung cancer screening: history, current perspectives, and future directions. Archives of medical science: AMS. 2015 Oct 12;11(5):1033. 
7. Gorycki1ABCDEF T, Lasek1BD I, Kamiński2CD K, Studniarek1DE M. Evaluation of radiation doses delivered in different chest CT protocols.

8. Kauczor HU, Bonomo L, Gaga M, Nackaerts K, Peled N, Prokop M, RemyJardin M, von Stackelberg O, Sculier JP, European Society of Radiology (ESR. ESR/ERS white paper on lung cancer screening. European radiology. 2015 Sep 1;25 (9):2519-31.

9. Kirchner J, Goltz JP, Lorenz F, Obermann A, Kirchner EM, Kickuth R. The "dirty chest"-correlations between chest radiography, multislice $\mathrm{CT}$ and tobacco burden. The British journal of radiology. 2014 Feb 13.

10. Blanchon T, Bréchot JM, Grenier PA, Ferretti GR, Lemarié E, Milleron B, Chagué D, Laurent F, Martinet Y, Beigelman-Aubry C, Blanchon F. Baseline results of the Depiscan study: a French randomized pilot trial of lung cancer screening comparing low dose CT scan (LDCT) and chest X-ray (CXR). Lung Cancer. 2007 Oct 31;58(1):508.

11. Villanti AC, Jiang Y, Abrams DB, Pyenson BS. A cost-utility analysis of lung cancer screening and the additional benefits of incorporating smoking cessation intervenetions. PloS one. 2013 Aug 7;8(8):e71379. 